\title{
Synthesis, characterization, and evaluation of paclitaxel loaded in six-arm star-shaped poly(lactic-co-glycolic acid)
}

This article was published in the following Dove Press journal:

International Journal of Nanomedicine

6 November 2013

Number of times this article has been viewed

\section{Yongxia Chen ${ }^{1,3, *}$ \\ Ziying Yang ${ }^{1, *}$ \\ Chao Liu' \\ Cuiwei Wang' \\ Shunxin Zhao ${ }^{2}$ \\ Jing Yang' \\ Hongfan Sun' \\ Zhengpu Zhang ${ }^{2}$ \\ Deling Kong' \\ Cunxian Song'}

'Tianjin Key Laboratory of Biomaterial Research, Institute of Biomedical Engineering, Chinese Academy of Medical Science and Peking Union Medical College, ${ }^{2}$ Institute of Polymer Chemistry, Nankai University, ${ }^{3}$ Center for Medical Device Evaluation of Tianjin, Tianjin, People's Republic of China

*These authors contributed equally to this work
Correspondence: Jing Yang 236 Baidi Road, Nankai District, Tianjin 300 I92, People's Republic of China Tel +862287890153

Fax +86228789 0153

Email yangjing37@hotmail.com
Background: Star-shaped polymers provide more terminal groups, and are promising for application in drug-delivery systems.

Methods: A new series of six-arm star-shaped poly(lactic-co-glycolic acid) (6-s-PLGA) was synthesized by ring-opening polymerization. The structure and properties of the 6-s-PLGA were characterized by carbon-13 nuclear magnetic resonance spectroscopy, infrared spectroscopy, gel permeation chromatography, and differential scanning calorimetry. Then, paclitaxel-loaded six-arm star-shaped poly(lactic-co-glycolic acid) nanoparticles (6-s-PLGA-PTX-NPs) were prepared under the conditions optimized by the orthogonal testing. High-performance liquid chromatography was used to analyze the nanoparticles' encapsulation efficiency and drug-loading capacity, dynamic light scattering was used to determine their size and size distribution, and transmission electron microscopy was used to evaluate their morphology. The release performance of the 6-s-PLGA-PTX-NPs in vitro and the cytostatic effect of 6-s-PLGA-PTX-NPs were investigated in comparison with paclitaxel-loaded linear poly(lactic-co-glycolic acid) nanoparticles (L-PLGA-PTX-NPs).

Results: The results of carbon-13 nuclear magnetic resonance spectroscopy and infrared spectroscopy suggest that the polymerization was successfully initiated by inositol and confirm the structure of 6-s-PLGA. The molecular weights of a series of 6-s-PLGAs had a ratio corresponding to the molar ratio of raw materials to initiator. Differential scanning calorimetry revealed that the 6-s-PLGA had a low glass transition temperature of $40^{\circ} \mathrm{C}-50^{\circ} \mathrm{C}$. The 6 -s-PLGA-PTX-NPs were monodispersed with an average diameter of $240.4 \pm 6.9 \mathrm{~nm}$ in water, which was further confirmed by transmission electron microscopy. The encapsulation efficiency of the 6-s-PLGAPTX-NPs was higher than that of the L-PLGA-PTX-NPs. In terms of the in vitro release of nanoparticles, paclitaxel (PTX) was released more slowly and more steadily from 6-s-PLGA than from linear poly(lactic-co-glycolic acid). In the cytostatic study, the 6-s-PLGA-PTX-NPs and L-PLGA-PTX-NPs were found to have a similar antiproliferative effect, which indicates durable efficacy due to the slower release of the PTX when loaded in 6-s-PLGA.

Conclusion: The results suggest that 6-s-PLGA may be promising for application in PTX delivery to enhance sustained antiproliferative therapy.

Keywords: PTX, polymer, drug delivery, nanoparticles, PLGA, antiproliferative therapy

\section{Introduction}

Poly(lactic-co-glycolic acid) (PLGA) is one of the commonly used drug-carrier matrices approved by the US Food and Drug Administration, and is widely used in biomedical applications due to its good biocompatibility and biodegradability. ${ }^{1,2}$ Several decades of biomaterials research have led to a progressively heightened interest in the use of PLGA-based nanoparticles (NPs) for drug delivery. For PLGA-based NPs, commercially 
available PLGA with a linear structure is widely accepted and used as a carrier matrix. However, much attention was initially focused on the modification of linear poly(lactic-coglycolic acid) nanoparticles (L-PLGA-NPs) to effectively meet a variety of requirements in drug delivery along with the need for treatment. For example, Subramanian et al modified the existing terminal carboxylic acid groups on the surface of PLGA NPs with p-aminobenzyl diethylenetriamine pentaacetic acid for enhanced availability of functional groups to make them suitable for technetium-99 m complexation. ${ }^{3}$ In this way, they improved both the radiolabeling yield and the biological efficacy of the NPs. Mittal et al optimized estradiol-loaded PLGA nanoparticulate formulations by varying the molecular weight and copolymer composition of PLGA, which resulted in the improved oral bioavailability and sustained release of estradiol. ${ }^{4}$ In another study, Stanwick et al designed a composite drug-delivery system comprised of neurotrophin-3 encapsulated in PLGA NPs dispersed in an injectable hydrogel to achieve sustained local delivery. ${ }^{5}$

However, as the technology and method of polymerization have advanced, polymer science research was no longer confined to the synthesis and modification of linear polymers. To improve the function of linear polymers and realize more complex functions in a single drug-delivery system, attention was transferred to adjusting the polymer structure. ${ }^{6-8}$ Nonlinear polymers with complex structures, including star-shaped polymers, dendrimers, and graft polymers, gradually become new research hotspots. Starshaped polymers, differing from linear analogs of identical molar weight, exhibit much better properties for use as carrier matrices. First, star-shaped polymers, which have a branched structure, have more arms, which are able to carry many more drug molecules than linear polymers. Moreover, star-shaped polymers have compact structures with smaller hydrodynamic volumes and radii of gyration. This means that they are not easily stranded in the blood, which ensures the bioavailability of the drugs encapsulated in them. ${ }^{9,10}$ Above all, star-shaped polymers provide more terminal groups that are capable of connecting with targeted molecules. These largely determine the properties of the polymer and transport the encapsulated drugs to the targeted organs and tissues. ${ }^{11-13}$

Currently, most star-shaped polymers in use are of three or four arms (Figure 1). However, the more arms the star-shaped polymer has, the more sites can be modified and the higher encapsulation efficiency it will have. In our study, we designed and synthesized a series of six-arm star-shaped PLGAs. Moreover, the methodology of orthogonal testing, which is
A

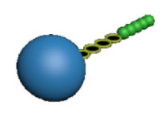

C

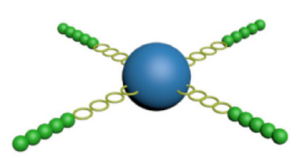

B

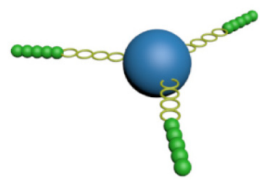

D

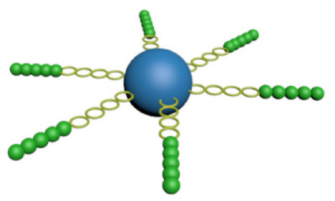

Figure I Schematic perspective view of (A) linear poly(lactic-co-glycolic acid) (PLGA), (B) three-arm star-shaped PLGA, (C) four-arm star-shaped PLGA, and (D) six-arm star-shaped PLGA.

based on orthogonality, was adopted to study multiple factors and levels in the experiment. To test part of the representative level combination chosen from a comprehensive selection and to reduce the workload, an orthogonal experiment was designed to find the optimal conditions for preparing paclitaxel-loaded six-arm star-shaped poly(lactic-co-glycolic acid) nanoparticles (6-s-PLGA-PTX-NPs). Following this, the obtained NPs were characterized and compared with paclitaxel-loaded linear poly(lactic-co-glycolic acid) nanoparticles (L-PLGA-PTX-NPs) in human aortic vascular smooth-muscle cells (T/G HA-VSMCs; American Type Culture Collection, Manassas, VA, USA).

\section{Materials and methods Materials}

$\mathrm{D}$, L-lactide (LA) with a melting point of $126.5^{\circ} \mathrm{C}-127.5^{\circ} \mathrm{C}$ and glycolide acid (GA) with a melting point of $83.5^{\circ} \mathrm{C}-85.5^{\circ} \mathrm{C}$ were procured from Glaco Ltd (Beijing, People's Republic of China). Inositol and stannous octoate $\left[\mathrm{Sn}(\mathrm{Oct})_{2}\right]$ were bought from the GuangFu Fine Chemical Research Institute (Tianjin, People's Republic of China). PTX was purchased from the Chongqing Meilian Company (Chongqing, People's Republic of China). L-PLGA (75/25, inherent viscosity $1.11 \mathrm{dL} \cdot \mathrm{g}^{-1}$ in chloroform $\left[\mathrm{CHCl}_{3}\right]$ at $30^{\circ} \mathrm{C}$ ) was bought from Birmingham Polymers, Inc (Birmingham, AL, USA). Polyvinyl alcohol (PVA) with a molecular weight (Mw) of 30,000-70,000 was obtained from Sigma-Aldrich (St Louis, MO, USA). Acetonitrile and methanol, used as the mobile phase in high-performance liquid chromatography (HPLC), and tetrahydrofuran of HPLC grade were purchased from Tianjin Concord Technology Co., Ltd. (Tianjin, People's Republic of China). All other chemicals were reagent grade or higher and were used without further purification. 


\section{Synthesis of six-arm star-shaped} poly(lactic-co-glycolic acid) $)_{x}\left(6-s-P L G A_{x}\right)$

The 6-s-PLGA formulations (where $\mathrm{x}=50,100,200$; representing the molar ratio of the raw materials to the initiator) were first synthesized by ring-opening polymerization of
LA and GA, with inositol as the multifunctional initiator and $\mathrm{Sn}(\mathrm{Oct})_{2}$ as the catalyst. The synthetic route is shown in Figure 2A. Briefly, for each 6-s-PLGA, the predetermined amount of LA, GA, and inositol were added into a clean, dry, and silanized polymerization tube in a nitrogen atmosphere.

A<smiles>O=C1COC(=O)CO1</smiles><smiles>CC1OC(=O)C(C)OC1=O</smiles><smiles>CCCC1C(O)C(O)C(O)C(O)C1O</smiles>

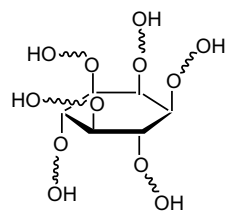

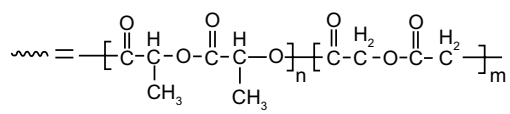

B
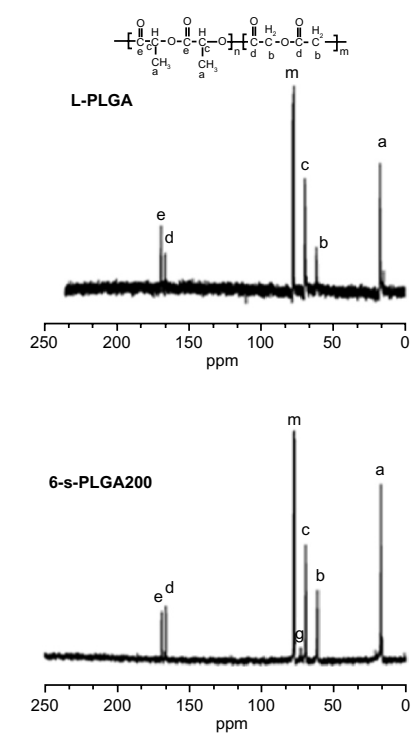

D

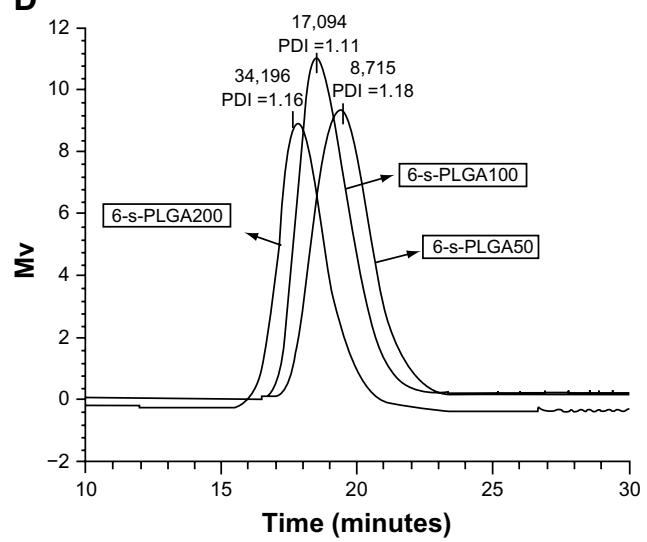

C

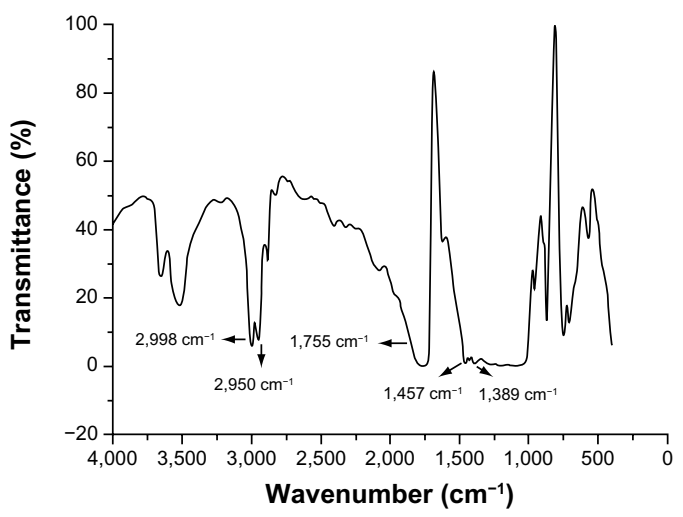

E

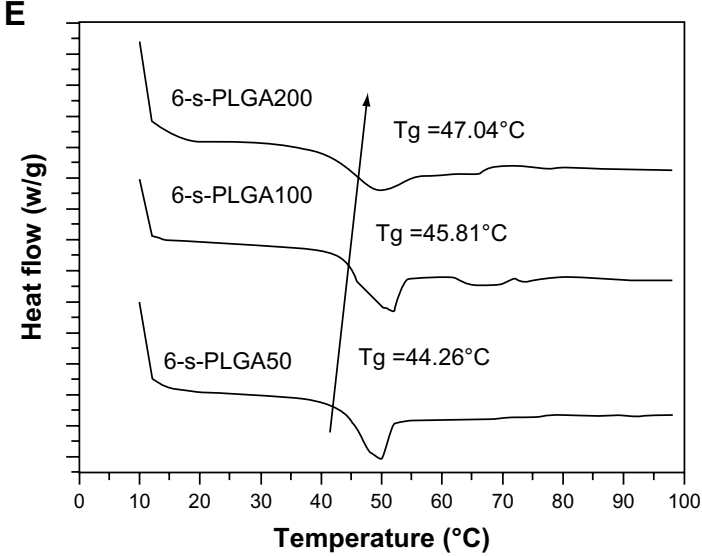

Figure 2 (A) Synthetic route of six-arm star-shaped poly(lactic-co-glycolic acid) (6-s-PLGA). (B) Carbon-13 nuclear magnetic resonance spectra of (a) linear poly(lactic-coglycolic acid) (L-PLGA) and (b) the synthesized 6-s-PLGA 200 with peak assignment. The 6-s-PLGA 200 exhibited a distinct resonance signal, "g." In comparison with L-PLGA, peak " $\mathrm{g}$ " was attributed to the -CH of inositol in the 6-s-PLGA 200 , indicating that the 6-s-PLGA was synthesized successfully. (C) Fourier-transform infrared spectrum clearly showing the five representative peaks of 6-s-PLGA. (D) Gel permeation chromatography spectrogram of the molecular weight and molecular weight distribution of 6-s-PLGA . The molecular weights of 6-s-PLGA ${ }_{50}, 6-\mathrm{s}-\mathrm{PLGA}_{100}$, and 6-s-PLGA 200 had a ratio of 1:2:4, which is basically in line with the molar ratio of the raw materials to initiator. (E) Glass transition temperature ( $\mathrm{Tg}$ ) of 6-s-PLGA with the heating rate of $5.0^{\circ} \mathrm{C} /$ minute. The $\mathrm{Tg}$ rose as the molecular weight of the polymer increased. Abbreviations: $\mathrm{PDI}$, polydispersity index; $\mathrm{Sn}(\mathrm{Oct})_{2}$, stannous octoate. 
A certain amount of $\mathrm{Sn}(\mathrm{Oct})_{2}$ with a molar ratio of $\mathrm{Sn}(\mathrm{Oct})_{2}$ to monomer of 1:10,000 was added and mixed in fully. The mixture was degassed under vacuum for 10 minutes then charged with nitrogen three times. Following this, another 30 minutes of degassing was required before the tube was sealed off. Finally, the polymerization was carried out in a vacuum oven at $160^{\circ} \mathrm{C}$ for 8 hours. Then, the rough product was dissolved in dichloromethane and purified by precipitation in a 20-fold excess of cold methanol. Each 6-s-PLGA was obtained by filtration and dried to constant weight in a vacuum oven at $40^{\circ} \mathrm{C}$.

\section{Characterization of 6-s-PLGA}

The structure and composition of each polymer was determined by carbon-13 nuclear magnetic resonance ( $\left.{ }^{13} \mathrm{C} N \mathrm{NM}\right)$ using a UNITY 400 spectrometer (Varian Inc, Palo Alto, CA, USA). The polymers were dissolved in deuterated dimethyl sulfoxide (DMSO-d6) and tetramethylsilane was used as the internal reference with a chemical shift of 0 ppm. The infrared spectra were recorded on a Nicolet ${ }^{\mathrm{TM}}$ Nexus 470-ESP Fourier-transform infrared (FTIR) spectrometer (Thermo Fisher Scientific, Waltham, MA, USA). The molecular weight and distribution of each polymer were measured by gel permeation chromatography using a Waters 510 pump (Milford, MA, USA): the sample concentration was $2.5-5.0 \mathrm{mg} / \mathrm{mL}$, chromatographically pure tetrahydrofuran was used as the mobile phase with a flow rate of $1.0 \mathrm{~mL} / \mathrm{minute}$, the standard reference substance was polystyrene of different molecular weights, and a differential refractive index detector was used. ${ }^{14}$ The glass transition temperature ( $\mathrm{Tg}$ ) of the polymer was measured by differential scanning calorimetry (DSC) using a Q2000 differential scanning calorimeter (TA Instruments, New Castle, DE, USA): a sample of 5-10 mg was heated from $0^{\circ} \mathrm{C}$ to $100^{\circ} \mathrm{C}$ in a nitrogen atmosphere $(50 \mathrm{~mL} /$ minute $)$ at a rate of $5.0^{\circ} \mathrm{C} /$ minute.

\section{Preparation of PTX-loaded NPs}

A solvent evaporation method, ${ }^{15,16}$ as previously reported, was adopted to prepare PTX-loaded NPs. In brief, 6-s-PLGA and PTX were dissolved in dichloromethane to form the organic phase, and subsequently injected into an aqueous solution containing PVA under constant sonication to form a stable emulsion. The solvent was then removed by continuous stirring at ambient temperature for several hours. Finally, the NPs were washed with distilled water three times to remove free PVA before being collected by centrifugation and dried by lyophilization.
L-PLGA-PTX-NPs were also prepared in the same way for use as the control.

\section{Orthogonal testing to determine the optimal conditions for the preparation of 6-s-PLGA-PTX-NPs}

Although the optimal conditions for preparing L-PLGA-NPs have been fully studied, ${ }^{17}$ little research has focused on the optimal conditions for preparing 6-s-PLGA-based nanoparticles (6-s-PLGA-NPs) using the solvent evaporation method. To obtain the optimal nanoparticulate formulation, L9 $\left(3^{4}\right)$ of orthogonal test was designed to explore the influence of the four factors involved in NP preparation - polymer concentration, ultrasonic time, PVA concentration, and the water and oil phase volume (W/O) - on the size and encapsulation efficiency of the NPs. ${ }^{18,19}$ Various levels of each factor are shown in Table 1.

\section{Characterization of the 6-s-PLGA-PTX-NPs}

We used 6-s-PLGA to prepare 6-s-PLGA-PTX-NPs under the optimal preparation conditions, which were based on the results of the orthogonal test. The amount of PTX in the NPs was determined by HPLC using an Agilent LC 1100 (Santa Clara, CA, USA): the NPs were completely dissolved in $2 \mathrm{~mL}$ dichloromethane, mixed with $4 \mathrm{~mL}$ mobile phase (acetonitrile/water, 50/50, v/v), then piped in a nitrogen atmosphere until the solution was clarified. The sample was fixed to $10 \mathrm{~mL}$ using filtered mobile phase. ${ }^{20,21}$ HPLC assay was performed using a reverse-phase diamond $\mathrm{C}_{18}$ column $(150.0 \times 4.6 \mathrm{~mm}$; Agilent Technologies, Santa Clara, CA, USA) and the chromatographic conditions were a column temperature of $40^{\circ} \mathrm{C}$, a mobile phase flow rate of $1.0 \mathrm{~mL} / \mathrm{minute}$, and the column effluent was detected using an ultraviolet detector at $\lambda_{\max }$ of $227 \mathrm{~nm}$. The size and size distribution were evaluated by dynamic light scattering on a Zetasizer NanoZS (Malvern Instruments Ltd, Malvern, UK). The NP dispersion was suitably diluted in deionized

Table I Factors and levels of orthogonal test design

\begin{tabular}{lllll}
\hline Level & \multicolumn{4}{l}{ Preparation factor } \\
\cline { 2 - 5 } & $\begin{array}{l}\text { Polymer } \\
\text { concentration } \\
(\mathbf{m g} / \mathbf{m L})\end{array}$ & $\begin{array}{l}\text { Ultrasonic } \\
\text { time } \\
\text { (minutes) }\end{array}$ & $\begin{array}{l}\text { PVA } \\
\text { concentration } \\
(\%)\end{array}$ & W/O \\
\hline 1 & 30 & 2 & 0.5 & $3: 1$ \\
2 & 50 & 5 & 1.0 & $6: 1$ \\
3 & 100 & 8 & 2.0 & $9: 1$ \\
\hline \multicolumn{4}{l}{ Abbreviations: PVA, polyvinyl alcohol; W/O, water and oil phase volume ratio. }
\end{tabular}


water at the optimal intensity (about $9 \times 10^{5}$ counts $/ \mathrm{s}$ ). Measurements were performed at $25^{\circ} \mathrm{C}$ on a $90^{\circ}$ angle. The zeta potential of the NPs was determined after diluting the sample in double-distilled water. The morphology of the prepared 6-s-PLGA-PTX-NPs was observed under transmission electron microscope (H-6009IV; Hitachi Ltd, Tokyo, Japan), with the NPs diluted with distilled water and placed on a copper grid. The sample was then dried at room temperature.

\section{In vitro stability of the 6-s-PLGA-PTX-NPs}

As aggregation of NPs may occur during storage and thus lead them to lose structural integrity or precipitate, it is necessary to examine the stability of NPs during storage. A certain amount of lyophilized powder of 6-s-PLGA-PTX-NPs and the formulation's corresponding aqueous dispersion were stored at $4^{\circ} \mathrm{C}$ in a static state. One month later, dynamic light scattering was used to measure the mean particle size of the 6-s-PLGA-PTX-NPs in aqueous dispersion, and HPLC was used to analyze the drug-loading capacity of the NPs in freeze-dried powder form. The particle size and drugloading capacity of the L-PLGA-PTX-NPs were also tested as the control. The results are expressed as mean \pm standard deviation $(n=3)$.

\section{Release of PTX from 6-s-PLGA-PTX-NPs}

The in vitro release behavior of PTX from 6-s-PLGA-PTXNPs and L-PLGA-PTX-NPs was investigated in $1.0 \mathrm{M}$ sodium salicylate solution at $37^{\circ} \mathrm{C}$ using double-chamber diffusion cells, as reported previously.22 Briefly, $5 \mathrm{mg}$ of the 6-s-PLGAPTX-NPs was added in $1.0 \mathrm{M}$ sodium salicylate solution $(5 \mathrm{~mL})$ then the NP dispersion was placed in one side of a double-chamber diffusion cell, while an equal volume of fresh sodium salicylate solution was added in the other side. The double-chamber diffusion cell was shaken at $280 \mathrm{rpm}$ at $37^{\circ} \mathrm{C}$ for 30 days. The collection fluid was changed with fresh medium at regular intervals to measure the PTX content, which was done following the same procedure and conditions as those outlined in the "Characterization of the 6-s-PLGA-PTX-NPs" section for the determination of drug-loading capacity. The in vitro release of PTX from the control L-PLGA-PTX-NPs was also determined. All tests were done in triplicate.

\section{In vitro biocompatibility analysis of synthesized 6-s-PLGA 200}

In this study, 6-s-PLGA was synthesized and preliminarily designed as a drug carrier in nanoparticulate form. Therefore, we examined the biocompatibility of blank
6-s-PLGA NPs. The biocompatibility of the blank 6-s-PLGA-NPs with T/G HA-VSMCs was assessed by 3-(4,5-dimethylthiazol-2-yl)-2,5-diphenyltetrazolium bromide (MTT) assay. Cells were grown at $37^{\circ} \mathrm{C}$ in a $5 \%$ (v/v) carbon dioxide atmosphere incubator in Gibco Dulbecco's Modified Eagle Medium/Nutrient Mixture F-12 (DMEM/F-12; Life Technologies, Carlsbad, CA, USA) supplemented with HyClone 10\% (v/v) fetal bovine serum (Thermo Fisher Scientific), and seeded in 96-well plates at a density of 5,000 viable cells per well to allow cell attachment after incubation overnight.

The cells were then exposed to a series of blank 6-s-PLGA-NP solutions of different NP concentrations ( 0.22 , 2.2 , and $22.0 \mu \mathrm{g} / \mathrm{mL}$ ) for a period of $1,2,3,4$, or 5 days. Blank L-PLGA-NP solutions with NP concentrations of $0.22,2.2$, and $22.0 \mu \mathrm{g} / \mathrm{mL}$ were used as controls. For the cytotoxicity assay, the medium was removed and the cells were washed with fresh phosphate-buffered saline. Following this, $180 \mu \mathrm{L}$ of DMEM/F-12, and $20 \mu \mathrm{L}$ of MTT solution $(5 \mathrm{mg} / \mathrm{mL}$ in phosphate-buffered saline) were added to each well and the plates were incubated for 4 hours. The MTT medium was removed and the formazan crystals were then dissolved with $150 \mu \mathrm{L}$ dimethyl sulfoxide (DMSO) at ambient temperature. $^{23,24}$ The optical density (OD) was measured at $570 \mathrm{~nm}$ with a Varioskan ${ }^{\circledR}$ Flash microplate reader (Thermo Fisher Scientific). The relative cell viability was calculated as:

$$
\text { Cell viability }(\%)=\frac{\mathrm{OD}_{\text {treat }}-\mathrm{OD}_{\text {blank }}}{\mathrm{OD}_{\text {control }}-\mathrm{OD}_{\text {blank }}} \times 100 \% \text {, }
$$

where "OD treated cells and control cells, respectively, and " $O D_{\text {blank }}$ " is the absorbance of the medium material, used for zero adjustment.

All tests were performed in quadruplicate. All these experiments were repeated at least three times.

\section{Proliferative inhibition of 6-s-PLGA- PTX-NPs on T/G HA-VSMCs}

Cytostatic assay of 6-s-PLGA-PTX-NPs and L-PLGAPTX-NPs was undertaken on T/G HA-VSMCs. Cells were exposed to a series of 6-s-PLGA-PTX-NP solutions at four PTX concentrations $(2.2,22.0,220.0$, and 2,200.0 ng/mL) for a regular incubation time (1, 2, 4, 6, or 7 days). Following this, the viability of the cells was evaluated by MTT assay as discussed in the "Release of PTX from 6-s-PLGA-PTXNPs" section. L-PLGA-PTX-NP solutions with the same PTX concentrations were used as control. 


\section{Statistical analysis}

Statistical analysis was carried out using SPSS (v 16.0; IBM Corp, Armonk, NY, USA). All results are expressed as mean \pm standard deviation. Groups of data were compared using analysis of variance followed by Dunnett's $t$-test. Values were considered statistically significant when $P<0.05$.

\section{Results and discussion}

\section{Characterization of 6-s-PLGA}

Star-shaped polymers are the simplest branched polymers because they have only one branched center. Long-chain branched polymers can be formed by chemically linking preformed polymers ("polymer first" method) or by growing polymer chains from a multifunctional initiator ("core first" method). ${ }^{25}$ In the core first method, initiators with cyclic architecture combined with branched polymers, such as biodegradable PLGA, have been used to increase the functionality of the star polymers. In the present study, 6-s-PLGA formulations were synthesized in only one step, and the polymers were confirmed to have the structure of branched macromolecules. The ${ }^{13} \mathrm{C}$ NMR spectra of the synthesized 6-s-PLGA 200 and commercially available L-PLGA along with the peak assignments are shown in Figure 2B. Peak "m" is the solvent, while we assigned peaks "a," "c," and "e" $(\delta=17.727,69.602$, and $169.025 \mathrm{ppm}$, respectively) to the $-\mathrm{CH}_{3},-\mathrm{CH}$, and $\mathrm{C}=\mathrm{O}$, respectively, of lactide. Peaks "b" and "d" $(\delta=61.363$ and $166.101 \mathrm{ppm}$, respectively) are the signals of $\mathrm{CH}_{2}$ and $\mathrm{C}=\mathrm{O}$, respectively, of glycolide. Compared with the signals in L-PLGA, peak "g" ( $\delta=73.105 \mathrm{ppm})$ was attributed to $-\mathrm{CH}$ of inositol in 6-s-PLGA, suggesting that inositol initiated the polymerization successfully. In addition, the integral ratio of the area of peak "e" and peak "d" is about $3: 1$, which is consistent with the presupposed block proportion of lactide and glycolide in the 6-s-PLGA .

FTIR spectrometry was used to evaluate the chemical structure of 6-s-PLGA. The FTIR spectra for 6-s- PLGA ${ }_{200}$, for example, is presented in Figure 2C. The characteristic peak of 6-s-PLGA at $1,755 \mathrm{~cm}^{-1}$ was due to the $\mathrm{C}=\mathrm{O}$ group, while the peaks at 2,998 and $2,950 \mathrm{~cm}^{-1}$ were attributed to the stretching vibration of $-\mathrm{CH}$, and the peaks at 1,457 and $1,389 \mathrm{~cm}^{-1}$ were attributed to the bending vibration of the $\mathrm{CH}$ and $\mathrm{CH}_{3}$ groups. Because the inositol content was extremely small, we did not see the peaks of its groups.

The molecular weight and molecular weight distribution of the 6-s-PLGA formulations are illustrated in Figure 2D. Molecular weights of 6-s-PLGA 50 , 6-s-PLGA 100 , and 6-s-PLGA 200 have a ratio of 1:2:4; this is basically in line with the molar ratio of raw materials to initiator. This suggests that the molar ratio of raw materials to initiator directly determine the molecular weight of the polymer; in other words, the molecular weight of a polymer could be well controlled by adjusting the molar ratio of monomer to initiator. The polydispersity index of all three kinds of polymer is $<1.2$, showing a unimodal distribution in Figure 2D.

The thermal properties of the 6-s-PLGA formulations were investigated by DSC. As shown in Figure 2E, the Tg of the 6-s-PLGA ${ }_{x}$ ranged from $40^{\circ} \mathrm{C}$ to $50^{\circ} \mathrm{C}$, which is higher than normothermia and suitable for drug carriers. Moreover, $\mathrm{Tg}$ of polymers with high molecular weights is higher than that of those with low molecular weights, because the mobility of the end segment is greater in the former than for the other segments in each chain; the ratio of the end segment increases as the molecular weight is lowered, which results in lowered $\mathrm{Tg} .{ }^{26}$

\section{Biocompatibility of 6-s-PLGA}

The biocompatibility of the commercially available L-PLGA has been widely recognized, so blank L-PLGA-NPs were used as a control group. The growth curves of T/G HAVSMCs exposed to a series of blank 6-s-PLGA-NP solutions at different NP concentrations (test groups) are illustrated in Figure 3. The cell viability in test groups did not change greatly as the concentration increased and high cell viability was maintained. Hence, the 6-s-PLGA-NPs had little toxicity on T/G HA-VSMCs, suggesting their favorable biocompatibility.

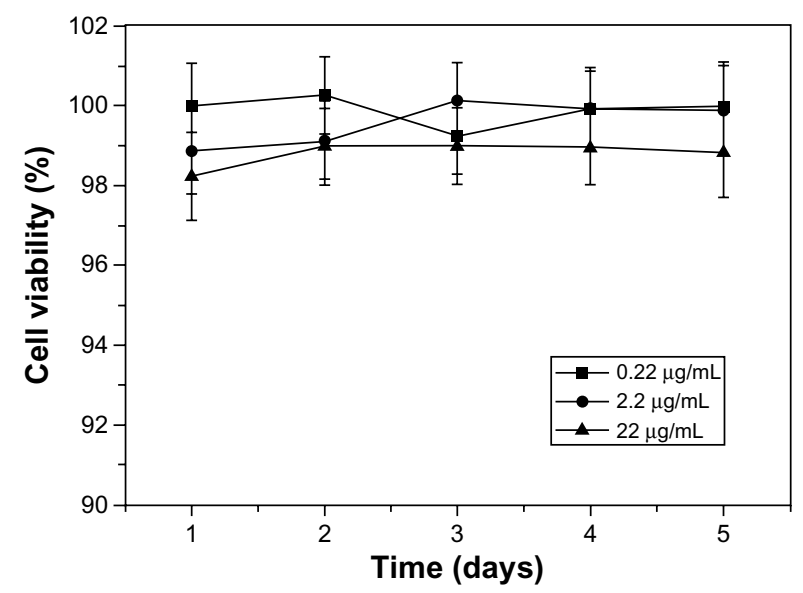

Figure 3 Cell growth of human aortic smooth-muscle cells incubated with blank paclitaxel-loaded six-arm star-shaped poly(lactic-co-glycolic acid) nanoparticles at three different polymer concentrations $(0.22,2.2$, and $22.0 \mu \mathrm{g} / \mathrm{mL}$ ). Blank nanoparticles based on linear poly(lactic-co-glycolic acid) (L-PLGA) solutions were used as controls.

Notes: In nanoparticle form, there was no significant difference between the six-arm star-shaped poly(lactic-co-glycolic acid) (6-s-PLGA) and the commercially available L-PLGA, suggesting the favorable biocompatibility of 6-s-PLGA. 


\section{Optimal conditions for preparing 6-s-PLGA-PTX-NPs}

PTX has been widely used in the treatment of cancer and cardiovascular disease. Taxol (Bristol-Myers Squibb, New York, NY, USA), the commercial form of PTX, is formulated in a vehicle composed of Kolliphor ${ }^{\circledR}$ EL, formerly known as Cremophor ${ }^{\circledR}$ EL (polyethoxylated castor oil; BASF SE, Ludwigshafen, Germany) and alcohol (50:50, v/v). This solvent has been associated with serious side effects, including severe hypersensitivity, nephrotoxicity, and neurotoxicity. ${ }^{27}$ NPs are one of the new strategies that have appeared in recent years to improve the solubility of PTX. For hydrophobic drugs, the solvent evaporation method is commonly used to prepare NPs. Since the optimal conditions for preparing L-PLGA-NPs have been fully studied, in this study we explored four key factors - polymer concentration, ultrasonic time, PVA concentration, and W/O - in the preparation of L-PLGA-NPs to obtain the optimal nanoparticulate 6-s-PLGA-NP formulation. ${ }^{28,29}$

According to the principle of orthogonal testing, the greater the value of the range, the greater the influence would be. The impact on particle sizes of the four factors studied is more obvious than that of encapsulation efficiency, as shown in Table 2. According to the results of statistical processing, there was no statistically significant effect of the four factors on the encapsulation efficiency of 6-s-PLGA-PTX-NPs. This is because in spite of the different preparation conditions, the PTX loading capacity of the 6-s-PLGA was not at maximum, which means more PTX molecules could be entrapped in the NPs due to the branched structure of 6-s-PLGA. With high drug-loading capacity, 6-s-PLGA-NPs may thus offer sustained drug delivery for the treatment of several diseases. However, the statistical data indicate that these four factors have an important influence on the particle size. Figure 4 clearly shows that polymer concentration $>\mathrm{W} / \mathrm{O}>$ ultrasonic time $>$ PVA concentration in terms of the degree of impact on the particle size. The particle size increased as the polymer concentration and $\mathrm{W} / \mathrm{O}$ increased. When the polymer concentration was $30 \mathrm{mg} / \mathrm{mL}$, the ultrasonic time was 8 minutes, PVA concentration was $1 \%$, and the $\mathrm{W} / \mathrm{O}$ was $3 / 1$, and the particle size of 6-s-PLGA-PTX-NPs was at its minimum - that is, the optimal nanoparticulate formulation of 6-s-PLGA-PTX-NPs with the minimum size was when the polymer concentration was $30 \mathrm{mg} / \mathrm{mL}$, there was 8 minutes of ultrasonic time, PVA concentration was $1 \%$, and the $\mathrm{W} / \mathrm{O}$ was $3 / 1$.

\section{Characterization of L-PLGA-PTX-NPs and 6-s-PLGA-PTX-NPs}

The L-PLGA-PTX-NPs and 6-s-PLGA-PTX-NPs, which were prepared under optimal preparation conditions (Figure 5A), exhibited high encapsulation efficiency and small particle size distribution, with a polydispersity index of $0.260 \pm 0.012$ and $0.262 \pm 0.017$, respectively, as shown in Table 3. For 6-s-PLGA-PTX-NPs, 95.7\%

Table 2 The influence of different polymer concentrations, ultrasonic times, polyvinyl alcohol (PVA) concentrations, and water and oil phase volume ratios (W/Os) on the size and encapsulation efficiency (EE) of paclitaxel-loaded six-arm star-shaped poly(lactic-coglycolic acid) nanoparticles

\begin{tabular}{|c|c|c|c|c|c|c|}
\hline \multirow[t]{2}{*}{ Number } & \multicolumn{4}{|l|}{ Preparation parameter } & \multicolumn{2}{|c|}{ Measurement index } \\
\hline & $\begin{array}{l}\text { Polymer concentration } \\
(\mathrm{mg} / \mathrm{mL})\end{array}$ & $\begin{array}{l}\text { Ultrasonic } \\
\text { time (minutes) }\end{array}$ & $\begin{array}{l}\text { PVA concentration } \\
\text { (\%) }\end{array}$ & W/O & Size (nm) & EE (\%) \\
\hline I & 30 & 2 & 0.5 & $3: 1$ & 312.4 & 98.1 \\
\hline 2 & 30 & 5 & I & $6: 1$ & 273.0 & 94.6 \\
\hline 3 & 30 & 8 & 2 & $9: 1$ & 288.4 & 86.3 \\
\hline 4 & 50 & 2 & I & $9: 1$ & 407.8 & 93.6 \\
\hline 5 & 50 & 5 & 2 & $3: 1$ & 347.5 & 92.7 \\
\hline 6 & 50 & 8 & 0.5 & $6: 1$ & 340.1 & 97.6 \\
\hline 7 & 100 & 2 & 2 & $6: 1$ & 445.1 & 97.5 \\
\hline 8 & 100 & 5 & 0.5 & $9: 1$ & 566.7 & 95.8 \\
\hline 9 & 100 & 8 & 1 & $3: 1$ & 240.4 & 95.7 \\
\hline RI & 291.267 & 388.433 & 406.400 & 300.100 & & \\
\hline $\mathrm{R} 2$ & 365.133 & 395.733 & 307.067 & 352.733 & & \\
\hline R3 & 417.400 & 289.633 & 360.333 & 420.967 & & \\
\hline Range (size) & 126.133 & 106.100 & 99.333 & 120.867 & & \\
\hline SI & 93.000 & 96.400 & 97.167 & 95.500 & & \\
\hline S2 & 94.633 & 94.367 & 94.633 & 95.567 & & \\
\hline S3 & 96.333 & 93.200 & 92.167 & 91.900 & & \\
\hline Range (EE) & 3.333 & 3.200 & 5.000 & 4.667 & & \\
\hline
\end{tabular}

Notes: $\mathrm{R}=$ average particle size at various levels for each factor; $\mathrm{S}=$ average $\mathrm{EE}$ at various levels for each factor. 

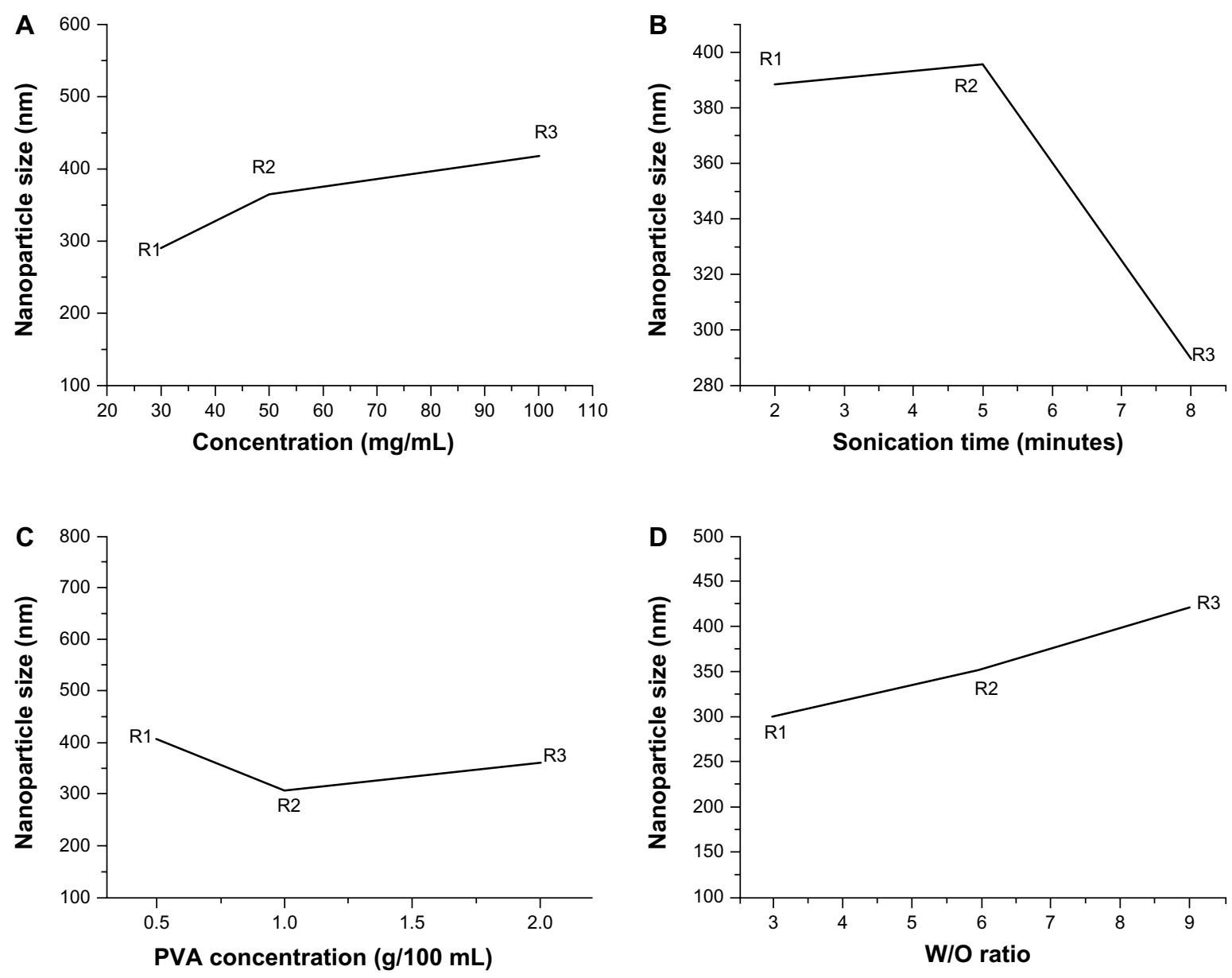

Figure 4 Effect curves of (A) polymer concentration, (B) ultrasonic time, (C) polyvinyl alcohol (PVA) concentration, and (D) water and oil phase volume ratio (W/O) on the particle size of paclitaxel-loaded six-arm star-shaped poly(lactic-co-glycolic acid) nanoparticles.

Notes: A polymer concentration of $30 \mathrm{mg} / \mathrm{mL}$, ultrasonic time of 8 minutes, PVA concentration of $1 \%$, and $\mathrm{W} / \mathrm{O}$ of $3 / \mathrm{I}$ were chosen as the optimal conditions when the particle size was at a minimum.

A

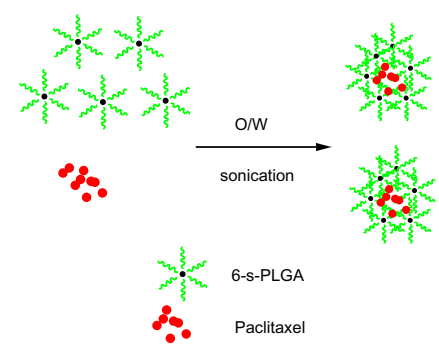

B

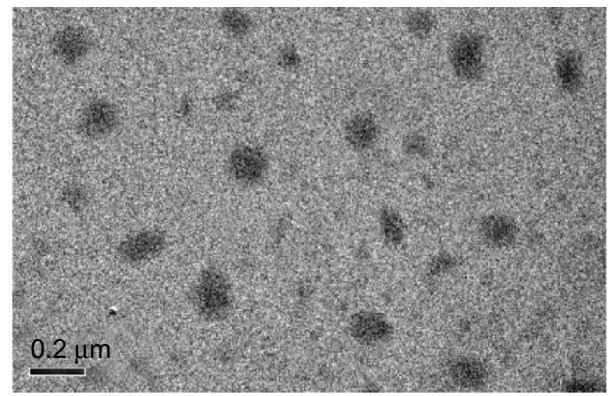

C

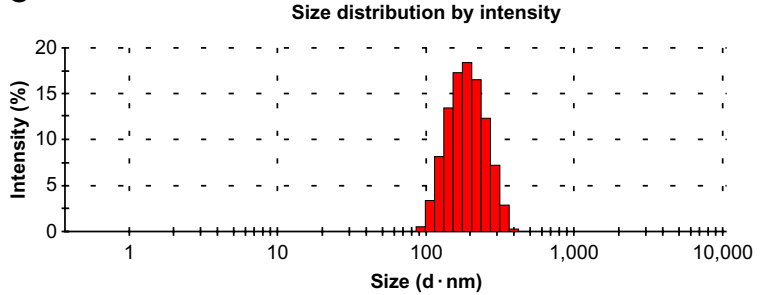

D

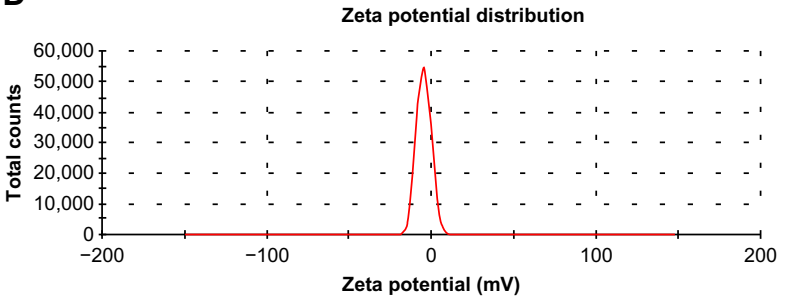

Figure 5 (A) Schematic diagram of the formation of paclitaxel-loaded six-arm star-shaped poly(lactic-co-glycolic acid) nanoparticles (6-s-PLGA-PTX-NPs). (B) Transmission electron microscopy image of 6-s-PLGA-PTX-NPs. (C) Particle size and size distribution. The 6-s-PLGA-PTX-NPs obtained had an average size of $240.4 \pm 6.9 \mathrm{~nm}$. (D) Zeta potential $(-4.5 \mathrm{I} \mathrm{mV})$ of the 6-s-PLGA-PTX-NPs.

Abbreviations: $\mathrm{W}$, water; $\mathrm{O}$, oil. 
Table 3 Characterization of paclitaxel-loaded six-arm star-shaped poly (lactic-co-glycolic acid) nanoparticles (6-s-PLGA-PTX-NPs) and paclitaxel-loaded linear poly(lactic-co-glycolic acid) nanoparticles (L-PTX-PLGA-NPs) (mean \pm standard deviation, $n=3$ )

\begin{tabular}{lllll}
\hline Sample & Size $(\mathrm{nm})$ & PDI & EE (\%) & DL (\%) \\
\hline L-PLGA- & $\mathbf{2 9 8 . 6} \pm 2.7$ & $0.260 \pm 0.012$ & $\mathbf{8 8 . 7} \pm 0.5$ & $\mathbf{8 . 6} \pm 0.7$ \\
PTX-NPs & & & & \\
$\begin{array}{l}\text { 6-s-PLGA- } \\
\text { PTX-NPs }\end{array}$ & $\mathbf{2 4 0 . 4 \pm 6 . 9}$ & $0.262 \pm 0.017$ & $\mathbf{9 5 . 7} \pm 0.3$ & $\mathbf{9 . 5} \pm 0.4$ \\
\hline
\end{tabular}

Note: Bold values indicate a significant reduction in particle size, a higher encapsulation efficiency and drug loading capacity of 6-s-PLGA-PTX-NPs compared to L-PLGA-PTX-NPs.

Abbreviations: DL, drug-loading capacity; EE, encapsulation efficiency; PDI, polydispersity index.

of the PTX was incorporated into the NPs, while the drug-loading capacity was significantly improved over that of the L-PLGAPTX-NPs. This can be attributed to the branched structure of 6-s-PLGA, which resulted in the entrapment of a greater amount of the drug. Transmission electron microscopy of 6-s-PLGAPTX-NPs (Figure 5B) further confirmed the favorable uniformity and narrow size range distribution of the NPs (Figure 5C). The zeta potential of the 6-s-PLGA-PTX-NPs was $-4.51 \mathrm{mV}$, as shown in Figure 5D. This demonstrated that there was plenty of net charge between particles, thus, the 6-s-PLGA-PTX-NPs was not easy to gather because of the big electrostatic repulsive force of the same charge. ${ }^{30,31}$

\section{Stability of L-PLGA-PTX-NPs and 6-s-PLGA-PTX-NPs}

Drug-loading capacity and particle size were used to evaluate the stability of the NPs. Lyophilized NP preparations are often required in industrial development, so, although the dispersion of NPs is mostly considered in research on NP stability, it is necessary to examine the stability of NPs in lyophilized powder form during storage. In our study, no obvious changes were observed in mean particle size in the aqueous dispersion of 6-s-PLGA-PTX-NPs or in the drug-loading capacity of the NPs lyophilized powder during storage, as shown in Figure 6. In the case of the L-PLGA-PTX-NPs, similar results with 6-s-PLGAPTX-NPs confirmed there was no aggregation when NPs were stored at $4^{\circ} \mathrm{C}$ for 1 month. This indicates that the prepared 6-s-PLGA-PTX-NPs had satisfactory stability and can be stored as either an aqueous solution or lyophilized powder at $4^{\circ} \mathrm{C}$ for a long time. This result demonstrates that storing 6-s-PLGAPTX-NPs will be convenient and safe for clinical applications and practical in terms of industrial development.

\section{In vitro PTX release}

The solubility of PTX in water is extremely low, so a special release medium must be chosen to achieve PTX's sink condition. Organic solvents and surfactants can be used as release mediums, but it is difficult to guarantee the stability of nanoparticles in an organic solvent. Following Huh et al, ${ }^{32}$ we chose $1.0 \mathrm{M}$ sodium salicylate solution as the release medium. The in vitro release of PTX from L-PLGAPTX-NPs and 6-s-PLGA-PTX-NPs in co-solvent sodium salicylate solution is illustrated in Figure 7. In comparison to the rapid action of the free drug, the release profile of the drug from the NPs is more complex; however, one of the major mechanisms is "drug diffusion," which means that the drug molecules are released slowly from the pores on the surface of the material, therefore, drugs can be released in a sustained manner to prolong the exposure time and improve the bioavailability of the drugs. ${ }^{33-36}$ As shown in Figure 7,
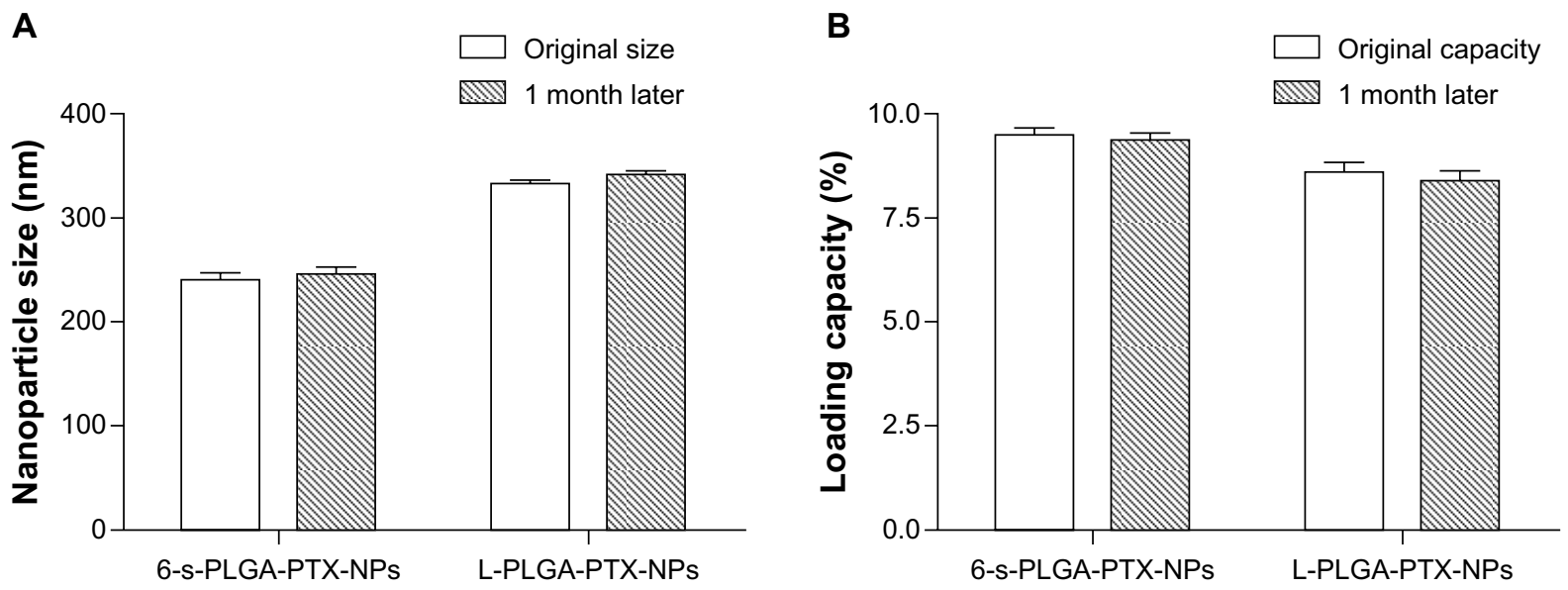

Figure 6 (A) Particle size and (B) paclitaxel loading capacity of paclitaxel-loaded six-arm star-shaped poly(lactic-co-glycolic acid) nanoparticles (6-s-PLGA-PTX-NPs) at baseline and after I month. Paclitaxel-loaded linear poly(lactic-co-glycolic acid) nanoparticles (L-PLGA-PTX-NPs) were tested as the control.

Notes: Bars represent mean \pm standard deviation $(n=3)$. No obvious change was observed in mean particle size or drug-loading capacity of the 6-s-PLGA-PTX-NPs during storage. This indicates the prepared 6-s-PLGA-PTX-NPs had satisfactory stability in both aqueous solution and lyophilized powder form at $4^{\circ} \mathrm{C}$. 


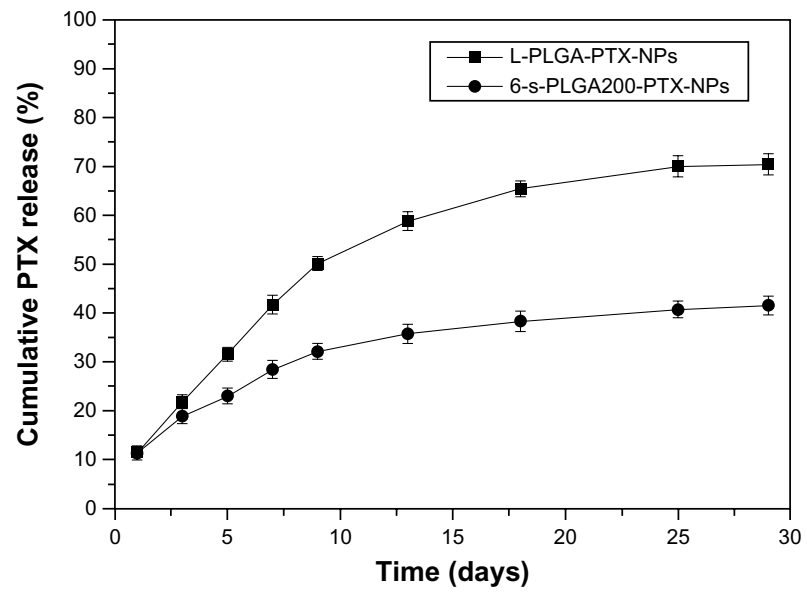

Figure 7 In vitro release profiles of paclitaxel (PTX) from paclitaxel-loaded sixarm star-shaped poly(lactic-co-glycolic acid) nanoparticles (6-s-PLGA-PTX-NPs) and paclitaxel-loaded linear poly(lactic-co-glycolic acid) nanoparticles (L-PLGA-PTX$\mathrm{NPs}$ ) in $1.0 \mathrm{M}$ sodium salicylate solution at $37^{\circ} \mathrm{C}$.

Notes: The 6-s-PLGA-PTX-NPs had a slower and more stable PTX release within 30 days than the L-PLGA-PTX-NPs, which exhibited complete and rapid PTX release in the same test period.

the cumulative amount of PTX released from the L-PLGAPTX-NPs and 6-s-PLGA-PTX-NPs on the first day was $10 \%$, followed by sustained release over the next 30 days, which indirectly demonstrates the polymer is suitable for using as a controlled-release carrier matrix. Moreover, compared with the more complete and more rapid drug release observed from L-PLGA-PTX-NPs, the dense structure of the 6-s-PLGAPTX-NPs hindered the dissolution and diffusion of PTX, resulting in more durable drug release. However, for some diseases, rapid drug release means better treatment. If this is necessary, additional pores can be created on the surface of the 6-s-PLGA-PTX-NPs using a pore-foaming agent to ensure rapid drug release.

\section{In vitro cytostatic effect of 6-s-PLGA-PTX-NPs}

To verify whether the released PTX was still pharmacologically active, and to evaluate the inhibition effects of 6-s-PLGA-PTX-NPs on cell division and proliferation for a long period, in vitro antiproliferative tests of 6-s-PLGA-PTXNPs against T/G HA-VSMCs were conducted. Pang et $\mathrm{al}^{37}$ previously observed that L-PLGA-PTX-NPs have a stronger sustained antiproliferative effect in comparison with Taxol. In the Taxol group, most cells were killed immediately, then, after 2 days, the remaining grew up. In the L-PLGA-PTX-NP group, although the cells were killed more slowly than in the Taxol group, cell toxicity appeared to be sustained for a long time. On the seventh day, all cells were killed. On the basis of this, and in the case of Taxol for which a significant effect was attributed to the excipient Kolliphor EL, L-PLGA-PTXNPs with the same PTX concentrations were chosen as the controls. The cytostatic effect of the L-PLGA-PTX-NPs and 6-s-PLGA-PTX-NPs was enhanced as the drug concentration was increased, as shown in Figure 8. For the L-PLGA-PTXNPs and 6-s-PLGA-PTX-NPs, the released PTX inhibited T/G HA-VSMCs in a sustained manner during the test period due to the slow release rate of PTX from the NPs. Moreover, it can be observed from Figure 8 that the 6-s-PLGA-PTXNPs and L-PLGA-PTX-NPs have a similar antiproliferative effect. However, since a slower PTX release was observed

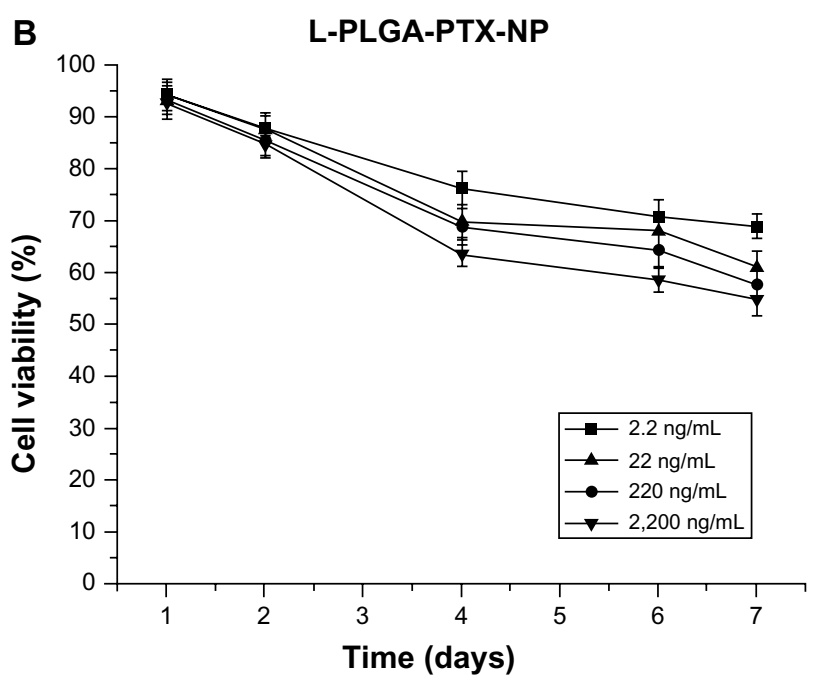

Figure 8 In vitro proliferative inhibitive effect of (A) paclitaxel-loaded six-arm star-shaped poly(lactic-co-glycolic acid) nanoparticles (6-s-PLGA-PTX-NPs) and (B) paclitaxelloaded linear poly(lactic-co-glycolic acid) nanoparticles (L-PLGA-PTX-NPs) at four different concentrations on human aortic smooth-muscle cells.

Notes: The cytostatic effect of paclitaxel (PTX) was dose dependent, and, in general, the 6-s-PLGA-PTX-NPs showed a similar antiproliferative effect to L-PLGA-PTX-NPs within 7 days. However, the 6-s-PLGA-PTX-NPs demonstrated a long-term antiproliferative effect and will require fewer administrations due to the slower release of PTX from six-arm star-shaped poly(lactic-co-glycolic acid). 
with 6-s-PLGA, 6-s-PLGA-PTX-NPs can achieve long-term efficacy. Further, the sustained drug release indicates that there would be a reduction in administration frequency, which would reduce patient suffering.

\section{Conclusion}

In this study, 6-s-PLGA was successfully synthesized by ring-opening polymerization. The molecular structure and properties of 6-s-PLGA were confirmed through ${ }^{13} \mathrm{C}$ NMR, FTIR, gel permeation chromatography, and DSC. The polymer has good biocompatibility and the 6-s-PTX-PLGA-NPs exhibited a smaller size distribution and higher drug-loading capacity than the L-PTX-PLGA-NPs. In vitro release and cytotoxicity studies of the 6-s-PTX-PLGA-NPs demonstrated a sustained and steady PTX release profile. The most important advantage of 6-s-PLGA is the high stability of the formed polyplexes. Thus, 6-s-PLGA may be a promising polymeric material for a PTX delivery vehicle in sustained antiproliferative therapy.

\section{Acknowledgments}

This work was supported by National Natural Science Foundation of China (81271706, 30800225), the Tianjin Natural Science Fund (13 JCZDJC30700), and the Research Institute of Ministry of Science and Technology (MOST) development projects (2012EG150136).

\section{Disclosure}

The authors report no conflicts of interest in this work.

\section{References}

1. Zou W, Cao G, Xi Y, Zhang N. New approach for local delivery of rapamycin by bioadhesive PLGA-carbopol nanoparticles. Drug Deliv. 2009;16(1):15-23.

2. Borselli C, Ungaro F, Oliviero O, et al. Bioactivation of collagen matrices through sustained VEGF release from PLGA microspheres. J Biomed Mater Res A. 2010;92(1):94-102.

3. Subramanian S, Pandey U, Gugulothu D, Patravale V, Samuel G. Modification of PLGA Nanoparticles for Improved Properties as a (99m) Tc-Labeled Agent in Sentinel Lymph Node Detection. Cancer Biother Radiopharm. 2013;28(8):598-606.

4. Mittal G, Sahana DK, Bhardwaj V, Ravi Kumar MN. Estradiol loaded PLGA nanoparticles for oral administration: effect of polymer molecular weight and copolymer composition on release behavior in vitro and in vivo. $J$ Control Release. 2007;119(1):77-85.

5. Stanwick JC, Baumann MD, Shoichet MS. Enhanced neurotrophin-3 bioactivity and release from a nanoparticle-loaded composite hydrogel. J Control Release. 2012;160(3):666-675.

6. Mooguee M, Omidi Y, Davaran S. Synthesis and in vitro release of adriamycin from star-shaped poly(lactide-co-glycolide) nano- and microparticles. J Pharm Sci. 2010;99(8):3389-3397.

7. Albertsson AC, Varma IK. Recent developments in ring opening polymerization of lactones for biomedical applications. Biomacromolecules. 2003;4(6):1466-1486.
8. Ober CK, Cheng SZ, Hammond PT, et al. Research in macromolecular science: challenges and opportunities for the next decade. Macromolecules. 2008;42(2):465-471.

9. Dechy-Cabaret O, Martin-Vaca B, Bourissou D. Controlled ring-opening polymerization of lactide and glycolide. Chem Rev. 2004;104(12): 6147-6176.

10. Dong CM, Qiu KY, Gu ZW, Feng XD. Synthesis of star-shaped poly(E-caprolactone)-b-poly(dl-lactic acid-alt-glycolic acid) with multifunctional initiator and stannous octoate catalyst. Macromolecules. 2001;34(14):4691-4696.

11. Qiu LY, Bae YH. Polymer architecture and drug delivery. Pharm Res. 2006;23(1):1-30.

12. McKee MG, Unal S, Wilkes GL, Long TE. Branched polyesters: recent advances in synthesis and performance. Prog Polym Sci. 2005;30(5):507-539.

13. Cai Q, Zhao Y, Bei J, Xi F, Wang S. Synthesis and properties of star-shaped polylactide attached to poly(amidoamine) dendrimer. Biomacromolecules. 2003;4(3):828-834.

14. Gao X, Wang BL, Wei XW, et al. Preparation, characterization and application of star-shaped PCL/PEG micelles for the delivery of doxorubicin in the treatment of colon cancer. Int J Nanomed. 2013;8: 971-982.

15. Varshosaz J, Soheili M. Production and in vitro characterization of lisinopril-loaded nanoparticles for the treatment of restenosis in stented coronary arteries. J Microencapsul. 2008;25(7):478-486.

16. Zhao T, Chen H, Dong Y, et al. Paclitaxel-loaded poly(glycolideco- $\varepsilon$-caprolactone)-b-D- $\alpha$-tocopheryl polyethylene glycol 2000 succinate nanoparticles for lung cancer therapy. Int $J$ Nanomed. 2013;8:1947-1957.

17. Gasper MM, Blanco D, Cruz ME, Alonso MJ. Formulation of L-asparaginase-loaded poly(lactide-co-glycolide) nanoparticles: influence of polymer properties on enzyme loading, activity and in vitro release. $J$ Control Release. 1998;52(1-2):53-62.

18. Johnson RA, Wichern DW. Applied Multivariate Statistical Analysis. 6th ed. Upper Saddle River, NJ: Prentice Hall; 2008.

19. Brandt S. Data Analysis: Statistical and Computational Methods for Scientists and Engineers. New York, NY: Springer-Verlag; 1999.

20. Hong X, Wei L, Ma L, et al. Novel preparation method for sustainedrelease PLGA microspheres using water-in-oil-in-hydrophilic-oil-inwater emulsion. Int J Nanomed. 2013;8:2433

21. Lopez-Gasco P, Iglesias I, Benedí J, et al. Characterization and in-vitro bioactivity evaluation of paclitaxel-loaded polyester nanoparticles. Anti-Cancer Drugs. 2012;23(9):947-958.

22. Thacharodi D, Rao KP. Development and in vitro evaluation of chitosan-based transdermal drug delivery systems for the controlled delivery of propranolol hydrochloride. Biomaterials. 1995;16(2): $145-148$.

23. Nakayama Y, Kakei C, Ishikawa A, et al. Synthesis and in vitro evaluation of novel star-shaped block copolymers (blocked star vectors) for efficient gene delivery. Bioconjugate chem. 2007;18(6):2037-2044.

24. Jiang L, Li X, Liu L, Zhang Q. Cellular uptake mechanism and intracellular fate of hydrophobically modified pullulan nanoparticles. Int J Nanomed. 2013;8:1825-1834.

25. Cloutet E, Fillaut JL, Astruc D, Gnanou Y. Star block copolymers and hexafullerene stars via derivatization of star-shaped polystyrenes. Macromolecules. 1999;32(4):1043-1054.

26. Zhao Y, Wang Z, Yang F. Characterization of poly(D,L-lactic acid) synthesized by direct melt polymerization and its application in Chinese traditional medicine compound prescription microspheres. $J$ Appl Polymer Sci. 2005;97(1):195-200.

27. Mu L, Feng SS. A novel controlled release formulation for the anticancer drug paclitaxel (Taxol): PLGA nanoparticles containing vitamin E TPGS. J Control Release. 2003;86(1):33-48.

28. Xie S, Wang S, Zhao B, Han C, Wang M, Zhou W. Effect of PLGA as a polymeric emulsifier on preparation of hydrophilic protein-loaded solid lipid nanoparticles. Colloids Surface B. 2008;67(2):199-204. 
29. Mainardes RM, Evangelista RC. PLGA nanoparticles containing praziquantel: effect of formulation variables on size distribution. Int J Pharm. 2005;290(1):137-144.

30. Feng S, Huang G. Effects of emulsifiers on the controlled release of paclitaxel (Taxol) from nanospheres of biodegradable polymers. J Control Release. 2001;71(1):53-69.

31. Costantino HR, Langer R, Klibanov AM. Aggregation of a lyophilized pharmaceutical protein, recombinant human albumin: effect of moisture and stabilization by excipients. Nat Biotechnol. 1995;13(5):493-496.

32. Huh KM, Lee SC, Cho YW, Lee J, Jeong JH, Park K. Hydrotropic polymer micelle system for delivery of paclitaxel. J Control Release. 2005;101(1-3):59-68.

33. Chen S, Pieper R, Webster DC, Singh J. Triblock copolymers: synthesis, characterization, and delivery of a model protein. Int J Pharm. 2005;288(2):207-218.

34. Nagahama K, Ohya Y, Ouchi T. Synthesis of star-shaped 8 arms poly(ethylene glycol)-poly(L-lactide) block copolymer and physicochemical properties of its solution cast film as soft biomaterial. Polym J. 2006;38(8):852-860.
35. Nagahama K, Saito T, Ouchi T, Ohya Y. Biodegradable Nano-aggregates of Star-Shaped 8-arm PEG-PLLA Block Co-polymers for Encapsulation of Water-Soluble Macromolecules. J Biomat Sci Polym Ed. 2011;22(1-3):407-416.

36. Duan Y, Zhang Y, Gong T, Zhang Z. Synthesis and characterization of MeO-PEG-PLGA-PEG-OMe copolymers as drug carriers and their degradation behavior in vitro. J Mater Sci Mater Med. 2007;18(10): 2067-2073.

37. Pang L, Wang H, Chen Y, Liu S, JingYang, Sun H. Paclitaxel polylactideco-glycolic acid nanoparticles inhibit human hepatocellular carcinoma cells HEPG2. Chinese Journal of Tissue Engineering Research 2013;17(3):412-418.
International Journal of Nanomedicine

\section{Publish your work in this journal}

The International Journal of Nanomedicine is an international, peerreviewed journal focusing on the application of nanotechnology in diagnostics, therapeutics, and drug delivery systems throughout the biomedical field. This journal is indexed on PubMed Central, MedLine, CAS, SciSearch $\AA$, Current Contents ${ }^{\circledR} /$ Clinical Medicine,

\section{Dovepress}

Journal Citation Reports/Science Edition, EMBase, Scopus and the Elsevier Bibliographic databases. The manuscript management system is completely online and includes a very quick and fair peer-review system, which is all easy to use. Visit http://www.dovepress.com/ testimonials.php to read real quotes from published authors. 